\title{
Acellular dermal matrix and bone cement sandwich technique for chest wall reconstruction
}

\author{
Chan Yeong $\mathrm{Heo}^{1}$, Byungkwon Kang ${ }^{1}$, Jae Hoon Jeong ${ }^{1}$, Kwhanmien Kim², Yujin Myung ${ }^{1}$ \\ Departments of ${ }^{1}$ Plastic and Reconstructive Surgery and ${ }^{2}$ Thoracic and Cardiovascular Surgery, Seoul National University Bundang Hospital, \\ Seoul National University College of Medicine, Seongnam, Korea
}

The authors performed rigid reconstruction using the sandwich technique for full-thickness chest wall defects by using two layers of acellular dermal matrix and bone cement. We assessed six patients who underwent chest wall reconstruction. Reconstruction was performed by sandwiching bone cement between two layers of acellular dermal matrix. In all patients, there was no defect of the overlying soft tissue, and primary closure was performed for external wounds. The average follow-up period was 4 years (range, 2-8 years). No major complications were noted. The sandwich technique can serve as an efficient and safe option for chest wall reconstruction.

Keywords Acellular dermis / Thoracic wall / Bone cements / Reconstructive surgical procedures / Thoracoplasty

\author{
Correspondence: Yujin Myung \\ Department of Plastic and \\ Reconstructive Surgery, Seoul \\ National University Bundang Hospital, \\ Seoul National University College of \\ Medicine, 82 Gumi-ro 173beon-gil, \\ Bundang-gu, Seongnam 13620, Korea \\ Tel: +82-31-787-7229 \\ Fax: +82-31-787-4055 \\ E-mail: surgene@snu.ac.kr
}

Received: June 9, $2021 \bullet$ Revised: August 9, $2021 \bullet$ Accepted: September 14, 2021

pISSN: 2234-6163 • elSSN: 2234-6171 • https://doi.org/10.5999/aps.2021.01067• Arch Plast Surg 2022;49:25-28

\section{INTRODUCTION}

The major indications of chest wall resection are tumors of the chest wall and lungs. Chest wall reconstruction is a challenging procedure as it involves rigid skeletal reconstruction and surrounding soft tissue coverage, in addition to resected tissue reconstruction, to prevent flail chest and subsequent respiratory distress [1]. In recent years, as adjuvant and neoadjuvant chemotherapy and radiotherapy options have evolved, thoracic surgeons have been able to operate more aggressively; thus, there is a growing need for efficient reconstruction methods [2].

Various prosthetic materials have been used for reconstruction. There are methods to reconstruct pleura and soft tissue using synthetic materials such as polypropylene and polyester [3] and to perform rigid fixation in skeletal reconstruction by sandwiching bone cement between these materials $[4,5]$. However, these methods have major disadvantages. Synthetic mesh is vul- nerable to postoperative infections in case of poor diffusion into the surrounding tissue, and direct interactions of polymethyl methacrylate with the remaining tissues above and below the synthetic mesh may cause foreign body reactions. Moreover, soft tissue flaps, bone grafts, or chimeric free flaps often require extensive surgery, and the possibility of various complications cannot be ruled out. Therefore, we replaced those synthetic materials and autologous flaps with acellular dermal matrix (ADM).

\section{IDEA}

We analyzed chest wall resection and reconstruction cases that were performed by the department of plastic and reconstructive surgery in consultation with the department of thoracic surgery from 2012 to 2018. Six patients (five men and one woman; mean age, 51 years) underwent chest wall reconstruction using ADM (AlloDerm, LifeCell Corp., Branchburg, NJ, USA) with 
the bone cement sandwich technique. We retrospectively analyzed all medical records, including radiographic images. In-
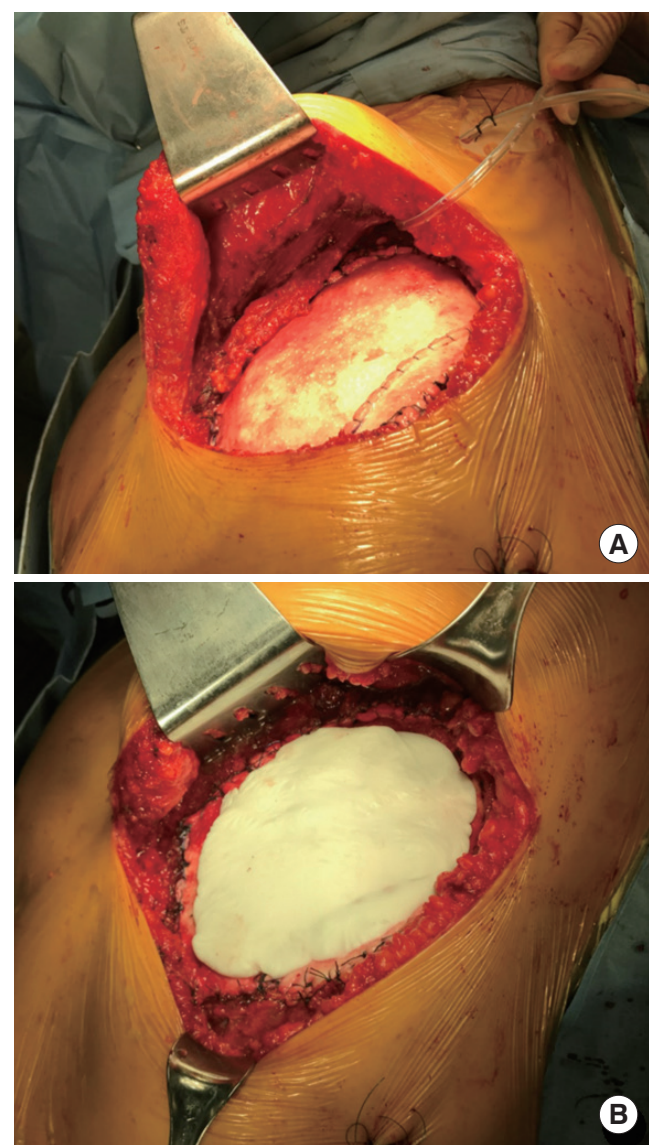

Fig. 1. Intraoperative photographs demonstrating the sandwich technique. (A) Acellular dermal matrix (ADM) was placed onto the Gore-Tex patch over the visceral pleura. (B) Bone cement was applied over the ADM layer.

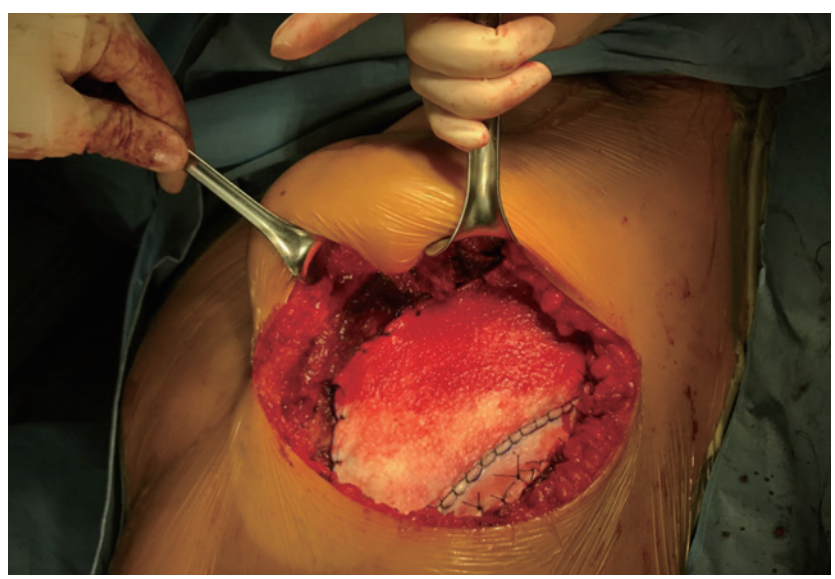

Fig. 2. Two layers of acellular dermal matrix (ADM) wrapping around the bone cement. The ADM prevented direct contact between the bone cement and the adjacent soft tissue. formed consent was obtained from the patients, including approval for photographic documentation.

Four patients had primary malignant tumors of the chest wall: three patients had chondrosarcoma, one had neurofibromatosis, and another had leiomyosarcoma. The other patient underwent resection of the chest wall combined with pneumonectomy for non-small cell lung cancer.

After the thoracic surgeon performed wide excision of the primary tumor, the visceral pleura was repaired with a Gore-Tex patch. The size of the ADM was selected to accurately cover the defect, the size of which depended on the extent of resection. First, the ADM was hydrated to cover the defect of the visceral pleura over the Gore-Tex patch, and reconstruction was performed with a $1-\mathrm{cm}$ margin over the border of the tissue defect (Fig. 1). Bone cement was then spread to cover the area where ribs were harvested. After complete hardening of the bone cement, reconstruction was completed by covering it with another layer of $\mathrm{ADM}$ (Fig. 2). Two layers of ADM were completely sutured in a water-tight fashion, establishing a pouch containing the bone cement, in order to prevent direct contact between the bone cement and adjacent soft tissue (Fig. 3). The patient was
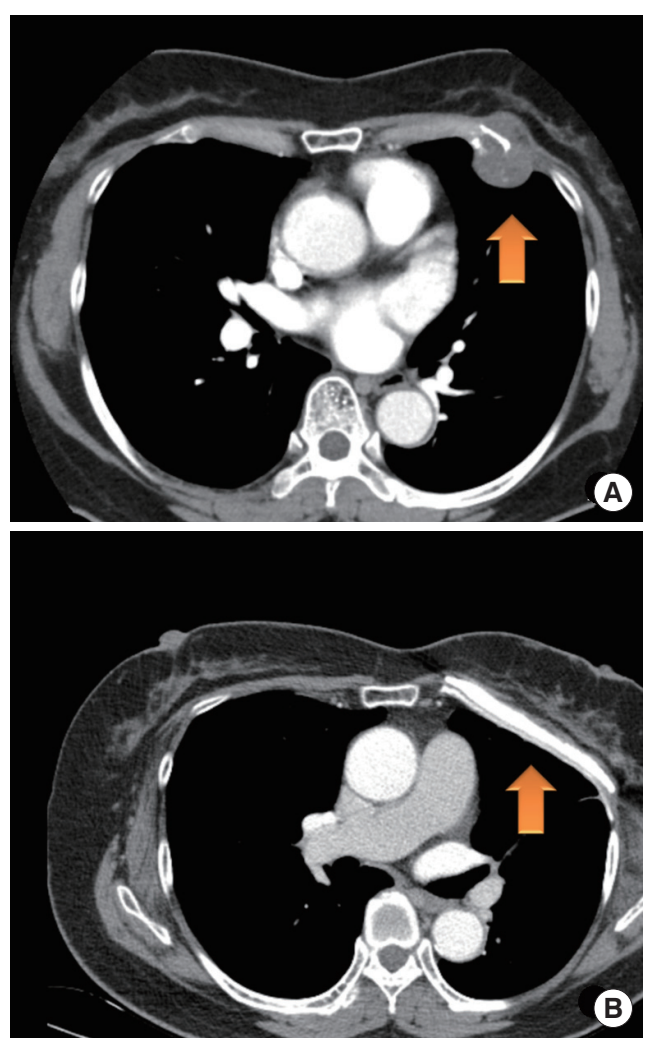

Fig. 3. Preoperative and postoperative computed tomography (CT) images showing the chest wall. (A) Preoperative CT shows chondrosarcoma on the patient's left fourth rib cartilage (arrow). (B) At 11 months after surgery, postoperative CT shows three layers of the reconstructed chest wall (arrow). 
checked for paradoxical breathing and natural movement of the chest wall, and the wound was closed in a layer-by-layer fashion with suction drainage in place.

Most of the patients were transferred to the general ward after 2 days of intensive care and were discharged 7.5 days (on average) later. During the average follow-up period of 19 months, the patients did not experience major complications such as postoperative wound infection, hemorrhage, respiratory complications (atelectasis, pleural effusion, and air leak), or arrhythmia.

\section{DISCUSSION}

Chest wall reconstruction is a challenging task for reconstructive surgeons due to the unique characteristics of the chest wall, which must exhibit mechanical capacities in order to facilitate respiratory movements, in addition to its core function as a mechanical barrier that separates the inner viscera from the outside space [1-3].

To date, methods to reconstruct the chest wall, including the pleura and muscular layers, have involved using synthetic materials and covering them with autologous flaps if needed [3-5]. Rigid prostheses have also been used for the reconstruction of defects of the rib cage. The most common method is mechanical barrier reconstruction using bone cement. However, this method has a critical disadvantage in that it is difficult to avoid foreign body reactions and potential donor site morbidity of the autologous flaps $[1,6,7]$.

In our cases, autologous flap surgery was not considered as the treatment of choice because sufficient skin and soft tissue remained present. Rigid reconstruction of the chest wall using non-stretchable materials may result in flail chest with paradoxical respiration, which can increase the respiratory burden. ADM is a more flexible material than those used in traditional fixation methods, such as bone cement, plates, or Prolene mesh. However, $\mathrm{ADM}$ itself cannot provide sufficient rigidity to protect the thoracic organs. In this regard, we propose the sandwich technique, wherein bone cement is added between $\mathrm{ADM}$ layers. This semi-rigid reconstruction technique is anatomically and functionally favorable and offers both firmness and flexibility of the chest wall.

ADM is highly biocompatible compared to Prolene mesh, which interacts with the recipient soft tissue and acts as a scaffold for donor-side cells after implantation to promote cell incorporation and revascularization $[8,9]$. It also has the advantage of a lower complication rate and significantly fewer foreign body reactions than occur with synthetic mesh. Moreover, by sealing the bone cement completely with a bioengineered, biocompatible skin product, it is possible to prevent bone cement from directly contacting adjacent soft tissues, thereby promoting safer tissue interactions.

We thought that the ADM should be thicker than that used for skin grafts because it should be able to protect against foreign body reactions in response to bone cement. It could also be of similar thickness or slightly thinner than that used for breast implant reconstruction. Therefore, the thickness of $\mathrm{ADM}$ in our technique was chosen to fall within that range (mean, $2.3 \mathrm{~mm}$; range, $1.5-3.0 \mathrm{~mm}$ ).

However, our approach involving the use of $\mathrm{ADM}$ is more expensive than those involving the use of synthetic mesh. The average amount of AlloDerm used was about $30 \times 30 \mathrm{~cm}$ per patient, which costs approximately $12,000,000$ Korean won $(\$ 10,000)$; this can be unaffordable for patients without full health insurance coverage. The patient's ability to bear these expenses must be discussed before surgery.

\section{NOTES}

\section{Conflict of interest}

No potential conflict of interest relevant to this article was reported.

\section{Ethical approval}

The study was approved by the Institutional Review Board of Seoul National University Bundang Hospital (IRB No. B-2009637-701) and performed in accordance with the principles of the Declaration of Helsinki.

\section{Patient consent}

The patients provided written informed consent for the publication and the use of their images.

\section{Author contribution}

Conceptualization: Y Myung. Data curation: B Kang, K Kim. Formal analysis: B Kang. Methodology: JH Jeong. Project administration: CY Heo. Visualization: B Kang. Writing-original draft: B Kang. Writing-review \& editing: Y Myung. All authors read and approved the final manuscript.

\section{ORCID}

Chan Yeong Heo https://orcid.org/0000-0001-9003-7365

Byungkwon Kang https://orcid.org/0000-0002-7704-0786

Jae Hoon Jeong https://orcid.org/0000-0002-4642-5560

Kwhanmien Kim https://orcid.org/0000-0002-6581-2750

Yujin Myung https://orcid.org/0000-0001-5051-2440 


\section{REFERENCES}

1. Netscher DT, Izaddoost S, Sandvall B. Complications, pitfalls, and outcomes after chest wall reconstruction. Semin Plast Surg 2011;25:86-97.

2. Basta MN, Fischer JP, Lotano VE, et al. The thoracoplastic approach to chest wall reconstruction: preliminary results of a multidisciplinary approach to minimize morbidity. Plast Reconstr Surg 2014;134:959e-967e.

3. Weyant MJ, Bains MS, Venkatraman E, et al. Results of chest wall resection and reconstruction with and without rigid prosthesis. Ann Thorac Surg 2006;81:279-85.

4. Collaud S, Pfofe D, Decurtins M, et al. Mesh-bone cement sandwich for sternal and sternoclavicular joint reconstruction. Eur J Cardiothorac Surg 2013;43:643-5.

5. Aghajanzadeh M, Alavi A, Aghajanzadeh G, et al. Recon- struction of chest wall using a two-layer prolene mesh and bone cement sandwich. Indian J Surg 2015;77:39-43.

6. Robinson TN, Clarke JH, Schoen J, et al. Major mesh-related complications following hernia repair: events reported to the Food and Drug Administration. Surg Endosc 2005; 19: 1556-60.

7. Wong RK, Gandolfi BM, St-Hilaire H, et al. Complications of hydroxyapatite bone cement in secondary pediatric craniofacial reconstruction. J Craniofac Surg 2011;22:247-51.

8. Ge PS, Imai TA, Aboulian A, et al. The use of human acellular dermal matrix for chest wall reconstruction. Ann Thorac Surg 2010;90:1799-804.

9. Sodha NR, Azoury SC, Sciortino C, et al. The use of acellular dermal matrices in chest wall reconstruction. Plast Reconstr Surg 2012;130(5 Suppl 2):175S-182S. 